\title{
Search for supersymmetry in events with a $Z$ boson, jets and missing energy using the JZB method
}

\author{
Marco - Andrea Buchmann for the CMS Collaboration* \\ Institute for Particle Physics, ETH Zürich, 8093 Zürich, Switzerlandn \\ E-mail: marco.andrea.buchmannecern.ch
}

\begin{abstract}
A search is presented for physics beyond the standard model (BSM) in events with a $Z$ boson, jets, and missing transverse energy $\left(E_{T}^{\text {miss }}\right)$. This signature is motivated by BSM physics scenarios, including supersymmetry. The study is performed using a sample of proton-proton collision data collected at $\sqrt{s}=7 \mathrm{TeV}$ with the CMS experiment at the LHC, corresponding to an integrated luminosity of $4.98 \mathrm{fb}^{-1}$. The contributions from the dominant standard model backgrounds are estimated from data using the jet-Z balance technique. In the absence of evidence for BSM physics, we interpret the results in the context of simplified model spectra.
\end{abstract}

36th International Conference on High Energy Physics,

July 4-11, 2012

Melbourne, Australia

${ }^{*}$ Speaker. 


\section{Introduction}

We describe a search [1] for Physics beyond the Standard Model (BSM) in a sample of pp collisions collected by the Compact Muon Solenoid (CMS) detector [2] at the Large Hadron Collider (LHC), at a center-of-mass energy of $7 \mathrm{TeV}$. The size of the data sample corresponds to $4.98 \mathrm{fb}^{-1}$. We search for final states with a $Z$ boson, jets and missing energy $\left(E_{T}^{\text {miss }}\right)$ where the $Z$ boson decays to electron or muon pairs.

The most significant Standard Model (SM) backgrounds in this final state are $Z+J$ Jets processes and $t \bar{t}$ production. The former is the most challenging since it peaks in the mass distribution of the two leptons and can have apparent missing energy due to detector resolution and reconstruction effects. After suppressing the Z+Jets contribution the latter becomes more important. To estimate the $t \bar{t}$ background we exploit lepton universality, which allows us to use a control sample of $e \mu$ events as well as events in the sidebands of the dilepton mass distribution.

We select events with two opposite sign leptons with $p_{T}>20 \mathrm{GeV}$. The dilepton system is required to have an invariant mass consistent with the mass of the $Z$ boson. We select jets with $p_{T}>30 \mathrm{GeV}$ and $|\eta|<3.0$.

\section{JZB Search}

The JZB variable is defined in the $x y$ plane as

$$
J Z B=\left|\sum_{j e t s} \vec{p}_{T}\right|-\left|\vec{p}_{T}^{Z}\right| \approx\left|-\vec{\not}_{T}-\vec{p}_{T}^{Z}\right|-\left|\vec{p}_{T}^{Z}\right|
$$

Thus JZB variable measures the imbalance between the $p_{T}$ of the reconstructed $\mathrm{Z}$ boson and that of the hadronic system. In SM Z+Jets events, the JZB distribution is approximately symmetric about zero, while for BSM physics it may be asymmetric, due to correlated production of the $\mathrm{Z}$ boson and invisible particles. Five signal regions are defined by requirements on the JZB event variable, from $\mathrm{JZB}>50 \mathrm{GeV}$ to $\mathrm{JZB}>250 \mathrm{GeV}$ in steps of $50 \mathrm{GeV}$. The signal region in the invariant mass distribution is defined as $\left|m_{l l}-m_{Z}\right|<20 \mathrm{GeV}$.

We search for BSM events where the $\mathrm{Z}$ boson is the decay product of a heavier (parent) particle of mass $m_{M}$ and is produced in conjunction with an undetectable decay product of mass $m_{\mathrm{X}}$, which gives rise to $E_{T}$. Let $p^{*}$ be the characteristic momentum of the decay products in the rest frame of the parent particle. If the parent particle has a mass of the order of the electroweak scale, $m_{M} \sim$ $O\left(m_{\mathrm{X}}+m_{\mathrm{Z}}\right), p^{*}$ is small, and $p^{*}$ can be smaller than the laboratory momentum of the parent. In that case, the daughter particles all appear in a tightly collimated angular region, the transverse momenta of the $\mathrm{Z}$ and invisible particle are balanced by the other particles in the decay chain, and large values of JZB can ensue. An example of such a decay chain is $\tilde{g} \rightarrow \bar{q}+\tilde{q} \rightarrow \bar{q}+q+\widetilde{\chi}_{2}^{0} \rightarrow \bar{q}+q+Z+\widetilde{\chi}_{1}^{0}$, where $\tilde{g}, \tilde{q}$, and $\widetilde{\chi}_{1,2}^{0}$ are the gluino, squark, and neutralino supersymmetric particles.

The balance between the jet system and the $\mathrm{Z}+\mathbb{E}_{T}$ system leads to large, positive JZB in events where $E_{T}$ and the $\mathrm{Z}$ boson are pair-produced. On the other hand, SM Z+Jets events populate the $\mathrm{JZB}>0$ and $\mathrm{JZB}<0$ regions evenly.

The principal SM backgrounds are divided in two categories. Backgrounds that produce oppositeflavor (OF) pairs $(e \mu, \mu e)$ as often as same-flavor (SF) pairs (ee, $\mu \mu)$ are referred to as "flavor- 
symmetric backgrounds". This category is dominated by $t \bar{t}$ processes. Backgrounds with two SF leptons from a $\mathrm{Z}$ boson are referred to as " $\mathrm{Z}$ boson backgrounds". This category is dominated by SM Z+Jets production.

Three non-overlapping data control regions are used to predict the contribution of flavor-symmetric backgrounds: (a) OF events compatible with the $\mathrm{Z}$ boson mass hypothesis (referred to as " $\mathrm{Z}$-peak region"), (b) OF events in the sideband of the Z boson mass peak, and (c) SF events in this sideband. The sideband region is defined as the union of $55<m_{l l}<70 \mathrm{GeV}$ and $112<m_{l l}<160 \mathrm{GeV}$. it is chosen so that it includes the same number of events as the Z-peak region in $t \bar{t}$ simulation. The two OF data control samples are compared in the region $30 \mathrm{GeV}<|\mathrm{JZB}|<50 \mathrm{GeV}$, which is outside the signal regions and has little contribution from signal or $Z \rightarrow \tau \tau+$ jets. The event yields from the two data control samples in this region are found to be in good agreement with each other and with expectations from the MC simulation.

The total contribution from flavor-symmetric backgrounds in the signal region is computed as the average of the yields in the three data control regions, as they provide independent estimates of the same background process.

SM backgrounds with a reconstructed $\mathrm{Z}$ boson are estimated using the negative JZB region after subtraction of flavor-symmetric backgrounds. This procedure relies on the fact that Z+Jets events with three or more jets evenly populate the negative and positive sides of the JZB distribution

Other backgrounds, though less significant, are also accounted for in these estimates. Contributions from the $\mathrm{SM} W Z$ and $Z Z$ processes are incorporated into the $Z+J$ ets estimate, since in these events the $Z_{T}$ and the $\mathrm{Z}$ boson candidates do not share the same parent particle. The background estimate from OF pairs accounts for $W W, Z \rightarrow \tau \tau$, and single-top production. Finally, events with one or more jets reconstructed as electrons or non-isolated leptons (from QCD multijet, $\gamma+$ jets, or electroweak processes) are accounted for by the background estimate from the sideband control regions.

The overall background prediction method is validated using a simulated sample including all SM backgrounds, with and without the inclusion of LM4 signal events. The comparison between the true and predicted distributions is shown in Fig. 1 for the two cases. We find that there is good agreement in the background-only case, while good sensitivity to a possible signal remains.

The comparison between the observed and predicted distributions is shown in Fig. 2. No significant excess is observed.

\section{Interpretation}

In the absence of a significant excess, we set upper limits on the production cross section of SMS models $[3,4,5]$, which represent decay chains of new particles that may occur in a wide variety of BSM physics scenarios, including SUSY. We provide cross section upper limits in the parameter space of these models, and exclude a region of the parameter space assuming reference cross sections and a $100 \%$ branching fraction to the final state under consideration (the $\mathrm{Z}$ boson is allowed to decay according to the well-known SM branching fractions).

Figure 3 illustrates the process considered in this study: two gluinos are produced, each of which decays to a pair of jets and the second-lightest neutralino $\widetilde{\chi}^{0}$, which itself decays to a $\mathrm{Z}$ boson and 

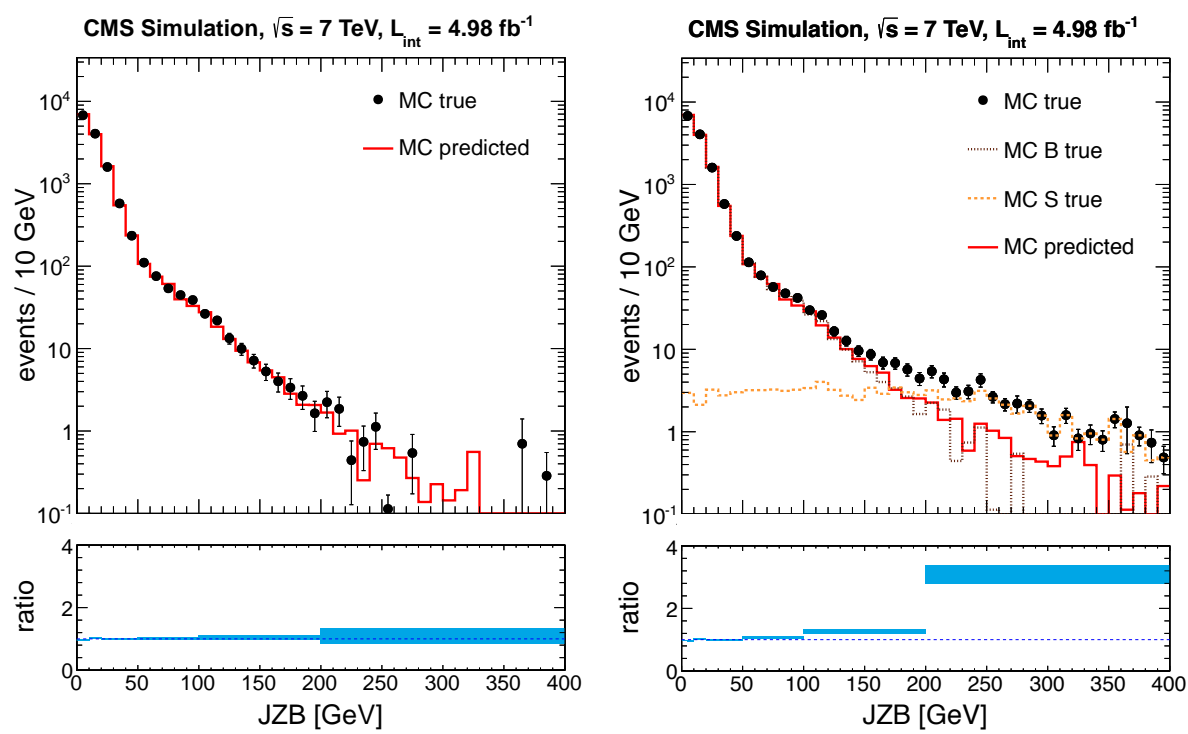

Figure 1: Comparison between true and predicted JZB distributions in the two cases: MC simulation without signal (left), MC simulation with signal (right). The lower plots show the ratio between observed and predicted distributions. The error bars on the observed distribution and in the ratio indicate the statistical uncertainty only.

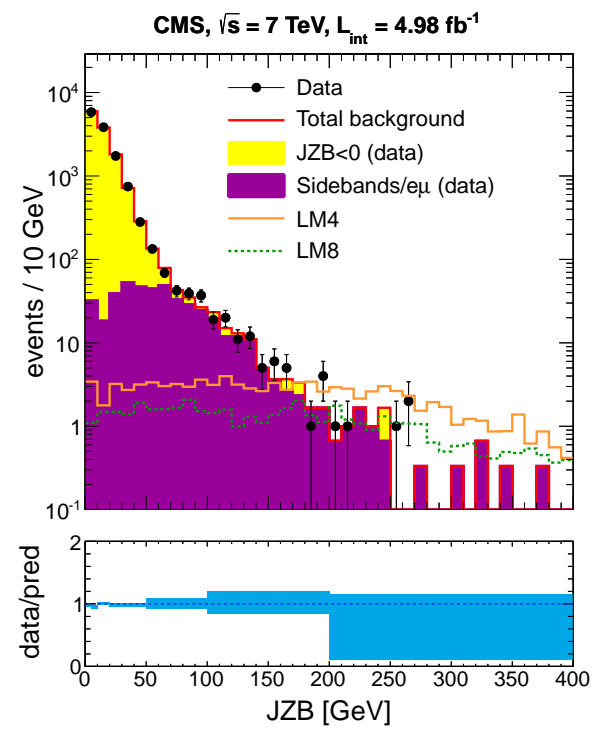

Figure 2: Comparison between the measured JZB distribution in the $\mathrm{JZB}>0$ region and that predicted from data control samples. The distribution from the LM4 MC is overlaid. The bottom plot shows the ratio between the observed and predicted distributions. The error bars indicate the statistical uncertainties in data only. 
the LSP $\widetilde{\chi}^{0}$. The parameters of the model are the masses of the gluino $\left(m_{\widetilde{g}}\right)$ and of the LSP $\left(m_{\widetilde{\chi}^{0}}\right)$. The mass of the intermediate neutralino $\left(m_{\widetilde{\chi}^{0}}\right)$ is fixed to $m_{\widetilde{\chi}_{2}^{0}}=m_{\widetilde{\chi}_{1}^{0}}+x \cdot\left(m_{\widetilde{g}}-m_{\widetilde{\chi}_{1}^{0}}\right)$, with $x=0.5$.

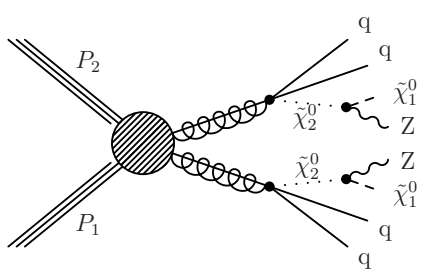

Figure 3: Simplified model for the production of two gluinos decaying into two $\mathrm{Z}$ bosons, two $\widetilde{\chi}_{1}^{0}$ particles, and jets.

To interpret these limits in terms of the gluino pair-production cross section, we use a reference cross section $\sigma^{N L O-Q C D}$ which corresponds to gluino pair-production in the limit of infinitely heavy squarks, calculated at NLO using PROSPINO [6] and the CTEQ6 [7] PDFs.

Figure 4 shows the $95 \%$ CL upper limits on the total gluino pair-production cross section.

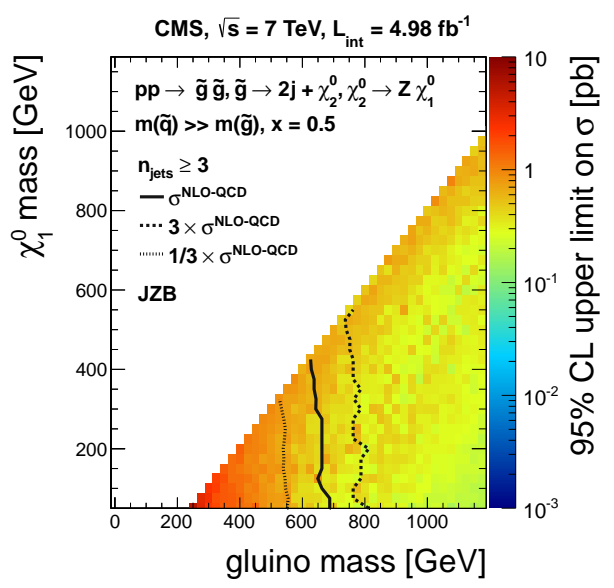

Figure 4: Limits on the SMS topology described in the text: 95\% CL upper limits on the total gluino pair-production cross section. The region to the left of the solid contour is excluded assuming that the gluino pair-production cross section is $\sigma^{N L O-Q C D}$, and that the branching fraction to this SMS topology is $100 \%$. The dotted and dashed contours indicate the excluded region when the cross section is varied by a factor of three. The signal contribution to the control regions is taken into account.

\section{Summary}

We have performed a search for BSM physics in final states with a leptonically-decaying Z boson, jets, and missing transverse energy. The jet- $Z$ balance method is used to suppress the dominant $Z+J$ ets background and to estimate the remaining background from data control samples. Backgrounds from $t \bar{t}$ processes are estimated using opposite-flavor lepton pairs and dilepton invariant mass sidebands. We find no evidence for anomalous yields beyond standard model (SM) expectations. The results are interpreted in the context of simplified model spectra. 


\section{References}

[1] CMS Collaboration, "Search for physics beyond the standard model in events with a Z boson, jets, and missing transverse energy in pp collisions at $\sqrt{s}=7$ TeV", Phys. Lett. B, 716 (2012) 260.

[2] CMS Collaboration, "CMS technical design report, volume II: Physics performance”, J. Phys. G, 34 (2007) 995.

[3] D. Alves et al. "Simplified Models for LHC New Physics Searches", (2011). arXiv: 1105.2838 .

[4] Nima Arkani-Hamed et al, "MARMOSET: The Path from LHC Data to the New Standard Model via On-Shell Effective Theories", (2007), arXiv: hep-ph/0703088.

[5] Bruce Knuteson and Stephen Mrenna, "BARD: Interpreting New Frontier Energy Collider Physics", (2006). arXiv:hep-ph/0602101.

[6] W. Beenakker, R. Hopker, and M. Spira, "PROSPINO: A Program for the Production of Supersymmetric Particles in Next-to-Leading Order QCD”, (1996). arXiv: hep-ph/9611232.

[7] J. Pumplin et al, "New Generation of Parton Distributions with Uncertainties from Global QCD Analysis", JHEP 07 (2002) 012. 